\title{
On computation of waveguide scattering matrices for the Maxwell system
}

\author{
B. A. Plamenevskii, A. S. Poretckii, and O.V. Sarafanov*
}

Statement of a boundary value problem. Let $G$ be a domain in $\mathbb{R}^{3}$ coinciding outside a large ball with the union of the non-overlapping semi-cylinders $\Pi_{+}^{q}=\left\{\left(y^{q}, t^{q}\right): y^{q} \in\right.$ $\left.\Omega^{q}, t^{q}>0\right\}$, where $q=1, \ldots, \mathcal{T}<\infty$ and $\Omega^{q}$ is a bounded domain in $\mathbb{R}^{2}$; the boundary $\partial G$ is supposed to be smooth. We consider the Maxwell system

$$
\begin{aligned}
i \operatorname{rot} u^{2}(x)-k u^{1}(x) & =0, \\
-i \operatorname{div} u^{2}(x) & =0, \\
-i \operatorname{rot} u^{1}(x)-k u^{2}(x) & =0, \\
i \operatorname{div} u^{1}(x) & =0
\end{aligned}
$$

in the domain $G$ with the boundary conditions

$$
\nu(x) \times u^{1}(x)=0,\left\langle u^{2}(x), \nu(x)\right\rangle=0, x \in \partial G,
$$

where $\langle\cdot, \cdot\rangle$ is the inner product in $\mathbb{C}^{3}, \cdot \times \cdot$ is the vector product in $\mathbb{R}^{3}$, and $\nu$ is the outward unit normal to $\partial G$. We set $U=\left(u^{1}, u^{2}\right)$ and write the system $(1),(2)$ in the form $\mathcal{M}(k) U=0$.

Continuous spectrum eigenfunctions. If for a number $k$ there exists a solution $U=$ $\left(u^{1}, u^{2}\right)$ to problem $(1),(2)$ that satisfies $U(x)=O(|x|)$ for $|x| \rightarrow \infty$ and does not belong to $L_{2}(G)$, we say that $k$ is a point of the continuous spectrum and $U$ is a continuous spectrum eigenfunction corresponding to $k$. It is known [2] that the continuous spectrum of problem (1), (2) coincides with the set $\left\{k \in \mathbb{R}:|k| \geq \mu_{\mathfrak{M}}^{1 / 2}\right\} \cup\{0\}$; here $\mu_{\mathfrak{M}}$ is the minimal positive eigenvalue among those of the Neumann problems for the Laplacian $-\Delta$ in the domains $\Omega_{q}$, $q=1, \ldots, \mathcal{T}$. The point $k=0$ formally belongs to the continuous spectrum, however, a scattering matrix is not defined at this point, therefore, we will not consider $k=0$ in what follows. A number $k$ is called an eigenvalue of problem (1), (2), if there exists a solution of the problem belonging to $L_{2}(G)$; such a solution is called an eigenfunction. The eigenvalues can not accumulate at finite distance and the eigenfunctions exponentially decay at infinity. We denote by $\operatorname{ker} \mathcal{M}(k)$ the eigenspace spanned by the eigenfunctions corresponding to an eigenvalue $k$. The space $\mathcal{E}(k)$, spanned by the continuous spectrum eigenfunctions corresponding to $k$, contains in particular the eigenspace ker $\mathcal{M}(k)$. The dimension of the quotient space $\mathcal{E}(k) / \operatorname{ker} \mathcal{M}(k)$ is called the multiplicity $\varkappa(k)$ of the continuous spectrum at

${ }^{*}$ The work was supported by grants of St.Petersburg University- 11.38.666.2013 and RFBR-12-01-00247a 
$k$. The number $\varkappa(k)$ is finite for all $k$, the function $k \rightarrow \varkappa(k)$ is even, piecewise constant, and increasing on the semiaxis $0<k<+\infty$; the discontinuity points of the function are called the thresholds. The positive thresholds form a sequence increasing to infinity. The value $\varkappa(k)$ remains constant on every interval between two neighboring thresholds. In what follows we consider $k$ on an interval $\left[k^{\prime}, k^{\prime \prime}\right]$ that is free of the thresholds.

Scattering matrix. In the space $\mathcal{E}(k) / \operatorname{ker} \mathcal{M}(k)$ there exists a basis with representatives $\left\{Y_{1}^{+}, \ldots, Y_{\varkappa}^{+}\right\}$such that

$$
Y_{j}^{+}(\cdot, k)=u_{j}^{+}(\cdot, k)+\sum_{l=1}^{\varkappa} s_{j l}(k) u_{l}^{-}(\cdot, k)+O\left(e^{-\delta|x|}\right), j=1, \ldots, \varkappa,
$$

for large $|x|$ and $\delta<\delta_{0}$, the number $\delta_{0}$ being sufficiently small; here $u_{j}^{+}\left(u_{j}^{-}\right)$are incoming (outgoing) waves. For large $|x|$ every wave satisfies (1) and (2) and on the set $\Pi_{+}^{q}$ for a certain $q$ and large $|x|$

$$
u_{j}^{ \pm}\left(y^{q}, t^{q} ; k\right)=\left|2 \lambda_{j}(k) k\right|^{-1 / 2} \exp \left(\mp i \lambda_{j}(k) t^{q}\right) \Phi_{j}^{\mp}\left(y^{q} ; k\right),
$$

while $u_{j}^{ \pm}(\cdot ; k)=0$ on $\Pi_{+}^{r}$ for $r \neq q$. The matrix $s(k)=\left\|s_{j l}(k)\right\|$ is independent of the choice of representatives in the mentioned basis. The matrix is unitary and is called the scattering matrix.

Elliptic extension of the Maxwell system. Let us introduce the operator $\{\mathcal{A}(D, k), \mathcal{B}\}$ of the elliptic boundary value problem in the domain $G$

$$
\begin{gathered}
\mathcal{A}(D, k) \mathcal{U}=\left(\begin{array}{r}
i \operatorname{rot} u^{2}+i \nabla a^{2}-k u^{1} \\
-i \operatorname{div} u^{2}-k a^{1} \\
-i \operatorname{rot} u^{1}-i \nabla a^{1}-k u^{2} \\
i \operatorname{div} u^{1}-k a^{2}
\end{array}\right), \quad x \in G, \\
\mathcal{B U}=\left(-\left\langle u^{1}, \tau_{2}\right\rangle,\left\langle u^{1}, \tau_{1}\right\rangle,\left\langle u^{2}, \nu\right\rangle, a^{2}\right), \quad x \in \partial G,
\end{gathered}
$$

where $\mathcal{U}=\left(u^{1}, a^{1}, u^{2}, a^{2}\right), u^{j}$ are three-component vector-valued functions, and $a^{j}$ are scalar functions, $j=1,2$. Here $\tau_{1}, \tau_{2}, \nu$ is a right-hand triplet of orthonormal vectors: $\tau_{1}$ and $\tau_{2}$ are tangent vectors, and $\nu$ is the outward normal to the boundary $\partial G$. The operator of this problem is self-adjoint with respect to the Green formula

$$
(\mathcal{A}(D, k) \mathcal{U}, \mathcal{V})_{G}+(\mathcal{B U}, \mathcal{Q V})_{\partial G}=(\mathcal{U}, \mathcal{A}(D, k) \mathcal{V})_{G}+(\mathcal{Q U}, \mathcal{B V})_{\partial G}
$$

where $\mathcal{U}$ and $\mathcal{V}$ are in $C_{c}^{\infty}\left(\bar{G} ; \mathbb{C}^{8}\right) ; \mathcal{Q U}=-i\left(\left\langle u^{2}, \tau_{1}\right\rangle,\left\langle u^{2}, \tau_{2}\right\rangle, a^{1},-\left\langle u^{1}, \nu\right\rangle\right)$ for $x \in \partial G$; finally, $(\cdot, \cdot)_{G}$ and $(\cdot, \cdot)_{\partial G}$ are the inner products in $L_{2}\left(G ; \mathbb{C}^{8}\right)$ and $L_{2}\left(\partial G ; \mathbb{C}^{4}\right)$, respectively. (In what follows we will not indicate the number of components in notations of the spaces of vectorvalued functions.) The boundary condition $-\left\langle u^{1}, \tau_{2}\right\rangle=\mathcal{G}^{1},\left\langle u^{1}, \tau_{1}\right\rangle=\mathcal{G}^{2}$ is equivalent to the equality $\nu \times u^{1}=\mathcal{G}^{1} \tau_{1}+\mathcal{G}^{2} \tau_{2}$ (see (2)).

Continuous spectrum eigenfunctions, waves, and a scattering matrix $S(k)$ can be introduced for the problem $\{\mathcal{A}(D, k), \mathcal{B}\} \mathcal{U}=0$ [1]. On the interval $k^{\prime} \leq k \leq k^{\prime \prime}$ the multiplicity $\Upsilon(k)$ of the continuous spectrum is constant, the scattering matrix $S(k)$ is block-diagonal, and one of its blocks coincides with $s(k)$ [2]. 
Auxiliary problem in truncated domain. We set $\Pi_{+}^{q, R}=\left\{\left(y^{q}, t^{q}\right) \in \Pi_{+}^{q}: t^{q}>R\right\}$ and $G^{R}=G \backslash \cup_{q=1}^{\mathcal{T}} \Pi_{+}^{q, R}$ for large $R$. Then $\partial G^{R} \backslash \partial G=\Gamma^{R}=\cup_{q=1}^{\mathcal{T}} \Gamma^{q, R}$, where $\Gamma^{q, R}=\left\{\left(y^{q}, t^{q}\right) \in\right.$ $\left.\Pi_{+}^{q}: t^{q}=R\right\}$. We introduce the elliptic boundary value problem

$$
\begin{array}{rlrl}
\mathcal{A}(D, k) \mathcal{U}(x) & =\mathcal{F}(x), & & x \in G^{R}, \\
\mathcal{B}(x) \mathcal{U}(x)=\mathcal{G}(x), & & x \in \partial G^{R} \backslash \Gamma^{R}, \\
(\mathcal{B}(x)+i \mathcal{Q}(x)) \mathcal{U}(x) & =\mathcal{H}(x), & & x \in \Gamma^{R} .
\end{array}
$$

The boundary $\partial G^{R}$ contains the edges $\partial \Gamma^{q, R}$. Let $\partial \Gamma^{R}=\cup_{q=1}^{\mathcal{T}} \partial \Gamma^{q, R}$ and let $r$ be a smooth positive function on $\overline{G^{R}} \backslash \partial \Gamma^{R}$ that coincides, near $\partial \Gamma^{R}$, with the distance dist $\left(x, \partial \Gamma^{R}\right)$. We denote by $H_{\beta}^{l}\left(G^{R}\right), l=0,1$, the completion of $C_{c}^{\infty}\left(\overline{G^{R}} \backslash \partial \Gamma^{R}\right)$ in the norm

$$
\left\|u ; H_{\beta}^{l}\left(G^{R}\right)\right\|=\left(\sum_{|\alpha| \leq l} \int_{G^{R}} r(x)^{2(\beta-l+|\alpha|)}\left|D_{x}^{\alpha} u(x)\right|^{2} d x\right)^{1 / 2}
$$

and by $H_{\beta}^{1 / 2}\left(\Gamma^{R}\right)$ and $H_{\beta}^{1 / 2}\left(\partial G^{R} \backslash \Gamma^{R}\right)$ the spaces of traces of the functions in $H_{\beta}^{1}\left(G^{R}\right)$ on $\Gamma^{R}$ and $\partial G^{R} \backslash \Gamma^{R}$, respectively.

Theorem 1. There exists a number $\varepsilon>0$ such that for $\beta \in(1 / 2-\varepsilon, 1 / 2+\varepsilon)$ and

$$
\{\mathcal{F}, \mathcal{G}, \mathcal{H}\} \in H_{\beta}^{0}\left(G^{R}\right) \times H_{\beta}^{1 / 2}\left(\partial G^{R} \backslash \Gamma^{R}\right) \times H_{\beta}^{1 / 2}\left(\Gamma^{R}\right)
$$

problem (4) has a unique solution $\mathcal{U} \in H_{\beta}^{1}\left(G^{R}\right)$. If $\mathcal{F}=0, \mathcal{G}=0$, and $\beta \leq 1 / 2$, the solution $\mathcal{U}$ satisfies $\left\|\mathcal{Q U} ; L_{2}\left(\Gamma^{R}\right)\right\| \leq\left\|\mathcal{H} ; L_{2}\left(\Gamma^{R}\right)\right\|$.

Functional and convergence theorem. Let $\mathcal{U}_{j}^{ \pm}, j=1, \ldots, \Upsilon$, be waves of problem $\{\mathcal{A}(D, k), \mathcal{B}\} \mathcal{U}=0$. We denote by $X_{l}^{R}(\cdot, k ; a)$ a solution to problem (4) with right-hand side

$$
\mathcal{F}=0, \quad \mathcal{G}=0, \quad \mathcal{H}=(\mathcal{B}+i \mathcal{Q})\left(\mathcal{U}_{l}^{+}(\cdot, k)+\sum_{j=1}^{\Upsilon} a_{j} \mathcal{U}_{j}^{-}(\cdot, k)\right)
$$

where $a=\left(a_{1}, \ldots, a_{\Upsilon}\right)$ is an arbitrary vector in $\mathbb{C}^{\Upsilon}$. Let us introduce the functional

$$
\mathbb{C}^{\Upsilon} \ni a \mapsto J_{l}^{R}(a ; k)=\left\|\mathcal{Q}\left(X_{l}^{R}(\cdot, k ; a)-\mathcal{U}_{l}^{+}(\cdot, k)-\sum_{j=1}^{\Upsilon} a_{j} \mathcal{U}_{j}^{-}(\cdot, k)\right) ; L_{2}\left(\Gamma^{R}\right)\right\|^{2} .
$$

Let $Z_{j, R}^{ \pm}$be a solution to problem (4) for $\mathcal{F}=0, \mathcal{G}=0$, and $\mathcal{H}=(\mathcal{B}+i \mathcal{Q}) \mathcal{U}_{j}^{ \pm}(\cdot, k)$. We have $X_{l}^{R}=Z_{l, R}^{+}+\sum a_{j} Z_{j, R}^{-}$. Now the functional takes the form

$$
J_{l}^{R}(a ; k)=\left\langle a E^{R}, a\right\rangle+2 \operatorname{Re}\left\langle F_{l}^{R}, a\right\rangle+G_{l}^{R},
$$

where $E^{R}$ and $F^{R}$ are matrices of size $\Upsilon \times \Upsilon$ with entries

$$
\begin{aligned}
& E_{i j}^{R}=\left(\mathcal{Q}\left(Z_{i, R}^{-}-\mathcal{U}_{i}^{-}\right), \mathcal{Q}\left(Z_{j, R}^{-}-\mathcal{U}_{j}^{-}\right)\right)_{\Gamma^{R}}, \\
& F_{i j}^{R}=\left(\mathcal{Q}\left(Z_{i, R}^{+}-\mathcal{U}_{i}^{+}\right), \mathcal{Q}\left(Z_{j, R}^{-}-\mathcal{U}_{j}^{-}\right)\right)_{\Gamma^{R}},
\end{aligned}
$$


$F_{l}^{R}$ is the $l$ th row of the matrix $F^{R}$, and $G_{l}^{R}=\left\|\mathcal{Q}\left(Z_{l, R}^{+}-\mathcal{U}_{l}^{+}\right)\right\|_{\Gamma^{R}}^{2}$.

As an approximation to the row $\left(S_{l 1}, \ldots, S_{l \Upsilon}\right)$ of the scattering matrix $S(k)$, we choose a minimizer $a^{0}(R, k)=\left(a_{1}^{0}(R, k), \ldots, a_{\Upsilon}^{0}(R, k)\right)$ of functional (5). From (6) it follows that $a^{0}(R, k)$ satisfies

$$
a^{0}(R, k) E^{R}+F_{l}^{R}=0
$$

consisting of such rows, the matrix $S^{R}$ serves as an approximation to the scattering matrix and satisfies $S^{R} E^{R}+F^{R}=0$.

Theorem 2. Let an interval $\left[k^{\prime}, k^{\prime \prime}\right]$ of the continuous spectrum of problem (1), (2) contain no thresholds. Then for all $k \in\left[k^{\prime}, k^{\prime \prime}\right]$ and $R>R_{0}$, where $R_{0}$ is a sufficiently large number, there exists a unique minimizer $a^{0}(R, k)$ of functional (5) and

$$
\left|a_{j}^{0}(R, k)-S_{l j}(k)\right| \leq C e^{-\delta R}, j=1, \ldots, \Upsilon
$$

here $\delta$ is the same as in (3) and the constant $C$ is independent of $k$ and $R$.

Since the matrix $s(k)$ is a block of the block-diagonal matrix $S(k)$, the corresponding block of $S^{R}(k)$ can be taken as an approximation to the matrix $s(k)$. The method can be modified for computing scattering matrices in a neighborhood of a threshold. Such a modification was described in [4] for the Helmholtz operator.

\section{References}

[1] S.A. Nazarov, B. A. Plamenevsky, "Elliptic problems in domains with piecewise smooth boundaries", de Gruyter Expositions in Mathematics, vol. 13, Walter de Gruyter \& Co., Berlin, 1994.

[2] B.A. Plamenevskii, A.S. Poretckii, "The Maxwell system in waveguides with several cylindrical ends", Algebra i Analiz, 25:1 (2013), 94-155; English Transl.: St. Petersburg Mathematical Journal, 25:1 (2014), 63-104.

[3] B.A. Plamenevskii, O.V. Sarafanov, "Method for computing waveguide scattering matrices in the presence of point spectrum", Funktsional'nyi Analiz i Ego Prilozheniya, 48:1 (2014), 61-72; English Transl.: Functional Analysis and Its Applications, 48:1 (2014), $49-58$.

[4] B.A. Plamenevskii, A.S. Poretckii, O.V. Sarafanov, "Method for computing waveguide scattering matrices in a neighborhood of thresholds", Algebra i Analiz, 26:1 (2014), 128164; English Transl.: St. Petersburg Mathematical Journal, 26:1 (2015), 91-116.

Translated by B.A. Plamenevskii 